A CASE

or

GASTROT O M Y

Fon

\title{
EXTRA-UTERINE GESTATION.
}

\author{
BY \\ JOHN ADAMS, F.R.C.S., \\ SURGEON TO THE LONDON HOSPITAL. \\ Received Sept. 29th.-Read Nov. 13th, 1860.
}

IN the early part of the spring of 1860 , I was requested by Dr. Ramsbotham to see a patient in the London Hospital with a large swelling in the abdomen, which he considered to be an extra-uterine fotus; and the history of the case led me to the same opinion.

Mary Jermy, æt. 28, of fair complexion and middle height, and with all the appearances of good health, had been married eight years. She had always menstruated at regular periods, but had never been pregnant before. In January, 1859, she menstruated very profusely, and very scantily in February; from March until May she was subject to occasional attacks of severe cramp-like pains, which were confined to the right side of the abdomen, extending from the pelvis to the hypochondrium: she felt very sick, but rarely vomited : she had no distinct morning sickness at any time. After February her menstrual secretion stopped, and she dated the commencement of her pregnancy from the early part of this month. In June vol. XLIV. 
she first felt the movements of a child, and her breasts perceptibly enlarged. This condition continued, and, after some time, she observed milk escaping from both breasts, more particularly the left; and the veins of the left leg and thigh became varicose.

She continued in good health, her abdomen increased in size, and she distinctly felt the movements of the child, until the 30th of October, a day or two prior to which she had a heavy fall, which was followed by soreness and cramp down to her knees. She considered herself in the ninth month of her pregnancy, and expected her confinement in the early part of November. She was visited by Mr. Williams, surgeon, of Plaistow, Essex, who was engaged to attend her. On the 30th of October she received a severe mental shock from her sister's death. At this time she ceased entirely to feel the movements of the child; and a week after this, she began to feel sleepy, tired, and worn, and suffered from a sense of stiffness in her limbs, but had no distinct pains like uterine pains. A discharge took place from the vagina, and blood, varying in colour from dark to pink, and pieces of flesh-like substance, entirely inoffensive in odour, were expelled in gushes. She reckoned her time of gestation to have terminated at the beginning of November. From this time she gradually diminished in size.

In January, 1860, she remained in much the same state. In February menstruation recommenced; and it has continued regularly ever sirce, varying in quantity, and usually expelled in gushes. The milk remained in her breasts until the following March, when I first saw her. Latterly she had become much thinner than she was.

On examination a hard oval tumour was felt, principally on the right side of the abdomen, extending from above the umbilicus to the right side of the symphysis pubis. There was a remarkable prominence in the tumour, which was quite immoveable, and very unlike any swelling from a fibrous tumour connected with the uterus. The hand could be readily passed around a great part of it, and the 
abdominal parietes could be made to glide indistinctly over its anterior surface. There was a feeling of irregularity about it; but I confess I could not distinctly recognise the individual portions of a feetus, for there was a good deal of subcutaneous fat in the walls of the abdomen. From my limited experience, I can hardly venture to assert that the sensation communicated to me by the examination was characteristic of this condition, but I recognised it at once as precisely similar to what $I$ had once witnessed in a parallel case. There was no pain on pressure. The uterus was found by examination per vayinam to be rather higher than usual, but there was no evidence of disease in it. She was able to perform her usual domestic duties, and expressed herself very anxious for the removal of the tumour.

There could be little or no doubt as to the precise nature of this case, and Dr. Ramsbotham and I agreed that the operation of gastrotomy should be performed; but we thought it prudent, on grounds hereafter to be stated, that this should be deferred until six months had elapsed from the end of what was supposed to be her natural gestation. She was readmitted in May, and, being then in perfect health, and fully alive to the risk of the operation, I performed the operation on the 31 st. She was readily placed under the influence of chloroform. I made an incision about five inches in length in a vertical direction over the most prominent part of the tumour, beginning a little above and on the right side of the umbilicus, and extending as far as within an inch and a half of the pubis. The integuments were divided, and I found that my incision had fallen on the sheath of the rectus. This was opened, and the muscle exposed; the peritoneum was opened to the same extent, and the surface of the tumour came into view, presenting a glistening aspect, and only slightly adherent at this part. The cyst, which was about four lines in thickness, and very firm, was opened, and a pint of a greenish-yellow, transparent fluid escaped, with yellowish flakes of vernix caseosa and some hairs. As soon as the cyst was opened, a loop of the funis protruded. The 
cyst was divided on the finger to the extent of the opening in the abdomen, and the funis being returned, and a portion of the rectus divided transversely, I felt the head and body of a fœtus, with its head uppermost, and its face towards the spine. I introduced my hand and seized an arm, which I pushed back, and then had no difficulty, owing to its limp state, in extracting the foetus by the breech. The funis was divided so as to leave about two inches outside the incision. On traction by the remaining portion of the funis, it was clearly ascertained that the placenta was firmly adherent, and, under the advice of Dr. Ramsbotham, no attempts were made to remove it. A piece of omentum, which had escaped through the opening, was cut away, and some rather large arteries, as well as some vessels in the cut edges of the cyst, were secured. I carefully sponged out all the fluid from the cyst, the walls of which collapsed, especially on the left side. Firm adhesions seemed to keep the right side in contact with the abdominal walls. The edges of the wound were carefully brought together by interrupted sutures, carried only through the integument and subjacent fat, and all the parts were kept in apposition by careful strapping, padding with cotton wool, and an elastic bandage.

Half a drachm of laudanum was given after the operation. She became exceedingly faint, but was restored at once by brandy. It would be useless to detail her progress with any degree of minuteness from day to day. She took moderate support, and a small quantity of brandy and good sherry, and she went on uninterruptedly well. The funis, which, on its first appearance during the operation, was thick and odematous, shrivelled up, and was altogether lost sight of on the fifth day after the operation; no doubt it escaped amongst the discharges. There remained for some time a small fistulous opening, with exuberant granulations, and a slight discharge of fetid matter at the lower part of the wound, where the funis had escaped.

October 1st.- Since this account was written, I have seen Mrs. Jermy three times. On her first visit she looked 
pale and ill, her legs were slightly swollen, and her general health was evidently suffering from some obscure cause. The fistulous opening still existed, and discharged a small quantity of fetid pus. I passed a steel director at least four inches towards the back of the pelvis, and this gave her pain. Caustic was applied to the orifice. Ten days or a fortnight after this she came to see me, and her health had materially improved; and about ten days ago she appeared quite well, and told me that the discharge had almost entirely ceased.

I subjoin an account of the fotus kindly sent me by Dr. Bader, the talented sub-curator of the Royal College of Surgeons.

October 2nd, 1860.- The length of the child from the top of the head to the soles of the feet was two feet; from the same point to the umbilicus ten and a half inches. The brain weighed three and a half ounces; the lungs one and a quarter ounce; the heart half an ounce; the liver one and a half ounce; the spleen one scruple; the intestines three ounces; weight of the child four pounds three ounces. The urachus and umbilical vessels were open. The lungs were pale, and floated freely. There was some yellow transparent fluid in the heart, together with several clots of pale-brown blood. The brain had a fleshy, red colour; its convolutions were developed; it had a smeary consistence. There was a great deal of meconium in the intestines. The child was a female, well developed. The cuticle peeled off in large flakes. There was no offensive odour. The head was well covered with fine, long, and light-brown hair. The nails were long and well developed. The parietal bones were slightly displaced, and overlapped by the occipital and frontal bones. Both corneæ were opaque; the eyes shrunk : no vitreous humour was found.

No opportunity was afforded in this case of proving the precise situation of the extra-uterine fotus; and whether it was developed in the walls of the uterus or in the abdominal cavity by attachment of the ovum to the peritoneal 
surface of the intestines, could not be made out; and, I need not say, this was not attempted. There are points, however, of serious importance in connection with this case, on which the practice pursued has a forcible bearing: these are, first, the propriety of performing any operation whatever under such circumstances; secondly, the time at which the operation should be performed, if thought desirable; and lastly, the method of performing it.

Are we justified in removing by operation an extrauterine fœtus, when nature affords us no indication of a desire to get rid of it by ulceration, pointing, \&c.? The question resolves itself into this-Is the risk incurred by the operation commensurate with that which the woman runs, if the case is allowed to remain intact? Let us first inquire what the risk is which is incurred by a female under such circumstances. Placing entirely out of consideration the propriety of non-interference during the evolution of the foetus, when it is quite clear that no. operation is justifiable, as all attempts to save the life of the child have been invariably fatal to the mother, is the chance that the fœetus may remain innocuous for an indefinite period to be weighed in the balance in favour of non-interference? I think it may, because there are on record a sufficient number of facts to influence the result. Many such cases are found compiled in Dr. William Campbelt's excellent memoir on extra-uterine gestation, published in 1840. He mentions nineteen cases in which the fotus remained in the abdomen of the mother during a period varying from ten to fifty-six years; and he refers to seven cases in which the mothers so circumstanced conceived once, two cases in which the mothers bore two children, four cases in which three children were born, two instances of five births, and one case where the mother gave birth to six children, whilst an extra-uterine fœtus remained in her abdomen. He makes, however, this judicious remark:- "Except in some rare cases, sooner or later, in consequence of many causes, which may or not be obvious, inflammation arises in the adjoining organs, involving the envelope of the fœtus." 
Such eases may fairly enter into our calculation in considering the propriety of operating, and they rather militate against any surgical interference during the quiescent state of the structures involved; but too much importance ought not to be attached to them.

In those cases which have remained innocent for so long a time, nature converts the cyst containing the fœtus into a material not readily acted on, which constitutes a permanent covering, as in the well-known case recorded by Mr. Lawrence, in the fifth volume of the 'Transactions' of this Society. The preparation is in the Pathological collection of the museum of the College of Surgeons, and is numbered 1722. In this instance calcification of the original cyst took place, and a bony case was formed around the fotus, which remained innocuous for fifty-two years, the woman dying of a disease quite irrespective of the original condition. Or the foetus undergoes a sort of petrifaction, by which it is converted into cartilage or bone, and is materially reduced in volume. Instances of this are referred to by Dr. Campbell.

Numerous instances are on record where the foetus has been got rid of by ulceration into the vagina, rectum, colon, bladder, as well as by abscess opening externally either at the umbilicus or some other part of the abdominal walls. It is most probable that, in all such cases, the decomposition and subsequent disintegration of the fœtus have preceded the efforts of nature to evacuate the contents of the cyst; for when the foetus has been extracted entire, however long a period may have elapsed after the termination of natural gestation, no decided evidences of putrefaction have been found. In a case quoted by Dr. Ramsbotham ("Principles and Practice of Obstetric Surgery'), where a fœus was removed by incision, which had evidently remained eight years in the abdomen of the mother, it was in "an astonishing state of preservation." The case is given in the 'Medico-Chirurgical Review' for 1834. A fistulous opening was formed at the umbilicus, which wast enlarged, and the fœetus was removed. In the case, also, the subject of the 
present paper, there were no decided evidences of putrefaction of the fœtus. It cannot for a moment be questioned that, where ulceration has indicated the point at which the fotus may be extracted, the removal, by surgical aid, may be accomplished generally with safety, and sometimes even with celerity; it nevertheless happens frequently that the mother dies a miserable death, being worn out by the constant discharge and low hectic fever. I have seen but two instances of extra-uterine gestation: one is the case now under consideration, and another I witnessed in consultation with Dr. Ramsbotham and Mr. Kennedy, of Stratford. In the latter case it was determined to wait until nature gave some indication, by pointing or by abscess, of her desire to evacuate the fotus; but at the very time when surgical interference was obviously called for, the patient declined any operation, and she sunk miserably, worn out by hectic and diarrhœa. I regretted that, in this case, I had not at an earlier period of the treatment advised the operation; for in consequence of the formation of a fluctuating tumour near the umbilicus, I was induced to introduce a trochar, and drew off a few ounces of serum : it is not improbable that this was really the indication I might have anticipated.

We have then, as militating against the operation of primary gastrotomy, the fact that the fœtus may remain innocuous for an indefinite period, and next that nature may expel the fotus by abscess or ulceration, at which time the surgeon's assistance may be frequently advantageously employed: in respect to the first, however, there is the prospect that, sooner or later, inflammation and its consequences may arise; and, in reference to the second, many mothers die during the efforts of nature to get rid of the incumbrance. Nevertheless, arguments deduced from a consideration of these circumstances might deter a timid surgeon from any interference, except under circumstances now so frequently mentioned; and it is quite clear that, if the spot where the incision should be made were always indicated, it would obviously be our duty to wait until this 
took place; but it must be admitted that frequently ulceration occurs in parts where it cannot at first be recognised, and where no surgical means can be employed to aid nature in her efforts.

The question which I propose for the consideration of the Society is-Are we justified in performing gastrotomy before nature affords us any indication of an attempt on her part to expel an extra-uterine fœtus? It is quite evident from recorded facts, that any attempt to remove a living child is invariably attended with a result fatal to the mother; and it is evident that no operation is justifiable until the fotus has become so far evolved that, from the situation, size, and character of the tumour, with an unimpeachable history from the mother, strengthened, if possible, by that of her medical attendant, not the slightest doubt can exist of its true character.

On this subject surgery affords us scarcely a sufficient number of facts to warrant a decided judgment. But are we to withhold an opinion on this ground, and to decline an operation because we have no statistical proof of its success? Certainly not, for this would at once strike at the root of all improvements in operative surgery. Let us look at the circumstances of the case, as afforded by this and other analogous instances. The fœtus is dead, and must sooner or later be felt as a foreign body, according to a natural law: if the operation by incision at an early period be not adopted, it must either be removed by the slow process of ulceration, or it must be so closed in as to be incapable of becoming a cause of further annoyance, except from its size and position. Of course, I am not anxious to evade the fact that gastrotomy may be successfully performed after ulceration has commenced. But the pointing may occur, as is often the case, in situations where no operation can be performed. I think that, even when no pointing has happened, as a foreign body it ought to be removed. So long, however, as it gives no inconvenience, the decision may rest entirely with the patient; but I certainly would not withhold my sanction to the operation, if she herself, on account 
of pain or other cause, were willing to rid herself of her burden.

To me there appear to be two circumstances of importance to be considered in regard to the question of primary gastrotomy, I mean where the operation is performed prior to pointing; namely, the period of evolution of the fotus, and the time at which the operation may be performed most safely after the termination of the life of the fotus. It is most probable that the older the fotus, or the nearer it has approached to maturity, the greater the chance of a successful result by primary operation; and, further, that vithin some limits, indefinite I admit, the longer the operation is deferred the better. But I refer both these circumstances to one and the same head, namely, the probability of adhesion having taken place between the cyst and the walls of the abdomen, so as to obviate one cause of danger-the risk of peritonitis.

In the case just related, the woman had begun to suffer uneasiness in the tumour, and was anxious that the foetus should be removed. Dr. Ramsbotham and I adopted the hint, but advised her to wait until six months had elapsed from the completion of her full period, at which time I anticipated a complete adhesion of the cyst to the peritoneum; in this I was to a certain extent disappointed. The operation was performed on the 31st of May, rather more than the six months, as it became necessary to aw.ait the termination of her catamenial period.

The only circumstance in connection with the operation requiring particular remarks-and I believe the observation refers to almost all cases of gastrotomy for extra-uterine gestation-relates to the placenta. From the few recorded cases of the operation, it appears that wherever any rude attempts have been made to extract the placenta, the cases have invariably been fatal. Nevertheless, it ought to be examined by very gentle traction of the funis, to see if it is loose, and can be removed with facility, otherwise it is better ta. leave it alone, with the hope that it will be separated and come away in the discharge; for while it remains, 
the wound will not close, and there is no doubt that the patient incurs the risk of pyæmia so long as the wound remains open.

Dr. Barnes, in the quarterly report on midwifery, contained in the fifty-second volume of the 'British and Foreign Medico-Chirurgical Review,' quotes a case of extra-uterine gestation operated on by Dr. Goodbrake, of Clinton, Illinois, United States, which terminated fatally. The case was that of a lady, forty-three years old, the mother of nine children, the youngest before her last pregnancy being about six years old. She became again pregnant, and was disappointed in the expectation of her confinement at the end of her full period. The tumour was on the right side, extending from above the umbilicus to the iliac fossa; it felt hard and irregular. There were other co-existent signs of pregnancy, which rendered the nature of the case quite clear. The patient was anxious to have an operation performed to get rid of the fœtus; this was done exactly two years and nine months from the end of her full period. The sac was traced to the right iliac fossa, to which, and to a considerable extent of the parietal peritoneum on the right side, it was firmly adherent. There were no adhesions anteriorly, nor to the intestines. A small incision was made into the sac, and a fotus was found in a good state of preservation. The cord was still attached to a very small placenta, of a cartilaginous character, seated low down in the pelvis, and immediately over the space where the sac was adherent to the broad ligament. The cord, and as much of the placenta and sac as could be got away without lacerating the peritoneum, were removed, the parts carefully sponged, and the incision brought together by the interrupted suture. The patient died on the fifth day, from prostration. I cannot conceive this violent procedure to be justifiable.

Postscript.-May 30th, 1861.-The wound, in the case of Mary Jermy, closed up at least four months ago; but she has suffered from a large ventral hernia, which is easily reduced, and is readily retained by a suitable truss. 